\section{Aspergillus laryngotracheobronchitis presenting as stridor in a patient with peripheral T cell lymphoma}

\author{
Ping-Hung Kuo, Li-Na Lee, Pan-Chyr \\ Yang, Yao-Chang Chen, Kwen-Tay Luh
}

\begin{abstract}
Invasive aspergillosis is a serious opportunistic infection in immunocompromised patients. The case history is described of a 44 year old patient with peripheral $T$ cell lymphoma who developed hoarseness and stridor after chemotherapy. Aspergillus fumigatus was isolated repeatedly from the sputum. Bronchoscopic examination showed symmetrical creamy-white exophytic lesions involving both vocal cords and the supraglottic area. There was diffuse tracheobronchitis with multiple raised cream-coloured plaques in the trachea which histologically consisted of numerous septate branching hyphae consistent with Aspergillus species. The lesions responded to systemic treatment with amphotericin $B$.

(Thorax 1996;51:869-870)
\end{abstract}

Keywords: stridor, aspergillosis, tracheobronchitis, larynx.

Fungal infection of the larynx is usually part of a widespread infection rather than an isolated process. ${ }^{1-4}$ It has been reported in patients treated with corticosteroids, with an underlying malignancy or immunodeficiency disease. The presenting symptom is usually hoarseness or dysphagia, but the presence of stridor is rare. ${ }^{4} \mathrm{We}$ report a patient with peripheral $\mathrm{T}$ cell lymphoma who presented with hoarseness and stridor due to Aspergillus laryngotracheobronchitis.

Department of Internal Medicine P-H Kuo

P-C Yang

Department of Laboratory Medicine

L-N Lee

Y-C Chen

K-T Luh

National Taiwan University Hospital, Taipei, Taiwan

Correspondence to: Dr K-T Luh.

Received 27 July 1995 Returned to authors 23 October 1995

Revised version received 14 November 1995

Accepted for publication

17 November 1995

\section{Case report}

A 44 year old man was admitted for modification of chemotherapy for peripheral $T$ cell lymphoma diagnosed 14 months earlier. His past medical history was otherwise unremarkable. On admission, respiratory examination and the chest radiograph were normal. Chemotherapy was given between the second and sixth day of admission and comprised etoposide, cytarabine, cisplatin and methylprednisolone ( $300 \mathrm{mg}$, days $2-5$ ). On day 8 he became febrile and developed a severe sore throat, hoarseness, and a brassy cough productive of whitish tenacious sputum. There was no evidence of sinusitis, oral thrush, or ulcers. Chest auscultation revealed mild inspiratory stridor and coarse breath sounds. The white blood cell count was $0.7 \times 10^{9} / 1$ with an absolute neutrophil count of $0.3 \times 10^{9} / 1$. The chest radiograph and computed tomographic scan were normal. Aspergillus fumigatus was isolated repeatedly from the sputum from day 9 onwards, and blood cultures were negative for bacteria and fungi. Broad spectrum antibiotics and granulocyte colony-stimulating factor were administered. His fever gradually subsided and the white blood cell count increased to $7.2 \times 10^{9} / 1$ on day 24 with an absolute neutrophil count of $4.5 \times 10^{9} / 1$. However, the hoarseness and productive cough persisted. The oxygen saturation of haemoglobin while breathing room air was $95 \%$. Bronchoscopic examination, performed on day 25 , disclosed the presence of creamy-white, exophytic lesions on the upper surface of both vocal cords and the supraglottic area in a symmetrical fashion (fig 1A). Mobility of both cords was impaired. The epiglottis was normal. Beyond the vocal cords there was diffuse tracheobronchitis with erythematous and oedematous mucosa. Multiple raised cream-coloured plaques were found throughout the tracheal mucosa. A single ulcer with overlying fibrinous exudate was also noted over the right main bronchus (fig 1B). There was no evidence of distal airway involvement. The exophytic lesions on both vocal cords were removed with the biopsy forceps as completely as possible. Both vocal cords were then found to have been partially destroyed with ulcers on their upper surface. Histologically, the tenacious plugs from both vocal cords consisted of numerous septate branching hyphae consistent with Aspergillus species, admixed with mucus and inflammatory cells. Systemic treatment with amphotericin B was introduced on day 26 at a dose of $1 \mathrm{mg} / \mathrm{kg}$ per day. The patient's cough and hoarseness improved gradually and stridor was no longer audible after two days of treatment. His voice had returned to almost normal one week later. Follow up bronchoscopy two weeks later showed that the lesions on the vocal cords and trachea had disappeared, with almost complete healing of the ulcer on the right main bronchus. Cytological examination of the bronchial washing fluid did not reveal any hyphal elements. Follow up fungal culture of the sputum was also negative. Because of severe systemic side effects amphotericin B was discontinued on day 45 at a cumulative dose of $1080 \mathrm{mg}$. Treatment was continued with oral itraconazole, $200 \mathrm{mg}$ twice daily. The patient's respiratory symptoms continued to improve and the follow up chest radiographs remained clear. Unfortunately, the patient had a relapse of his lymphoma and exacerbation of the haemophagocytic syndrome. Chemotherapy was resumed under cover of strong antibiotics but the patient died on the 53rd hospital day from septic shock.

\section{Discussion}

Aspergillus has now emerged as the leading cause of death from infectious fungal organisms in immunocompromised hosts. ${ }^{5}$ About $90 \%$ of the invasive aspergillosis involves the upper and lower respiratory tracts. ${ }^{6}$ The lung parenchyma is usually affected. Aspergillus tracheobronchitis, with infection limited prin- 

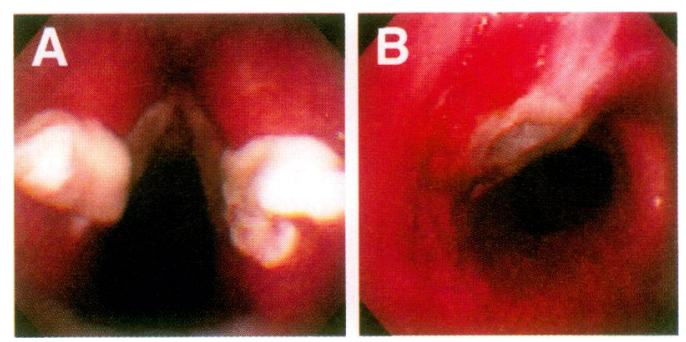

Figure 1 (A) Bronchoscopic examination showing creamy-white exophytic lesions over both vocal cords and supraglottic areas in a symmetrical fashion. (B) Appearance of the right main bronchus demonstrating bronchitis with a single ulceration and overlying fibrinous exudate.

cipally to the larger airways, is an uncommon manifestation. The disease was seen in only 15 $(6.9 \%)$ of 217 cases of intrathoracic aspergillosis, $^{7}$ and has been reported in both transplant and non-transplant immunocompromised patients. Sputum plugs can be filled with hyphae and are usually culture positive for Aspergillus. Airway obstruction may be rapidly progressive because of enlarging aspergillomas, ${ }^{7}$ a situation tantamount to multiple foreign bodies in the airway. ${ }^{6}$ Death occurs as a result of direct intrathoracic invasion and subsequent dissemination or, less commonly, from airway obstruction due to mucoid impaction or pseudomembrane formation. $^{7}$

Bronchoscopic examination may reveal varying degrees of erythema and oedema of the airway mucosa, with one to multiple ulcerative lesions, occasionally with exophytic nodules or masses. ${ }^{78} \mathrm{~A}$ less common form of infection is local plaque formation with elements of peribronchial invasion and microabscesses. ${ }^{8}$

The larynx is an unusual site of mycotic infection, either as a primary lesion or as a secondary focus. Reported pathogens have included blastomyces, histoplasma, coccidioides, Candida and Aspergillus species. ${ }^{1-4}$ Aspergillus infection of the larynx is surprisingly uncommon; indeed, none of the 98 patients with systemic aspergillosis studied by Young et al had had laryngeal involvement. ${ }^{9}$ Primary aspergillosis of the larynx simulating carcinoma has been reported. ${ }^{2}$ Depending on the degree of respiratory obstruction, stridor may or may not be present, but hoarseness and loss of voice power are almost universal findings. ${ }^{1}$ The most striking feature in this case was the symmetrical compact masses formed by tangled Aspergillus mycelia on both vocal cords. Fibreoptic bronchoscopy has allowed evaluation of this unusual manifestation which could previously be studied only at necropsy. Removal of the vocal cord lesions during biopsy may also facilitate the relief of symptoms in these patients.

The optimal treatment of Aspergillus tracheobronchitis remains problematic. Early diagnosis is crucial to prevent further dissemination of disease. ${ }^{4}$ Antifungal agents may be helpful in treating patients with normal immune systems, but their value in treating immunocompromised patients is uncertain. Amphotericin B is still considered as a first-line agent for this infection. ${ }^{3}$ In the case presented here the airway lesions responded well to this drug, although it was associated with severe systemic side effects. Aerosolised and liposomal amphotericin B offer hope of higher, more effective dosing with less toxicity. The new antifungal agent itraconazole is also promising. ${ }^{10}$ Surgery is recommended for mechanical complications such as persistent haemoptysis or airway obstruction. In view of the dramatic therapeutic response in this case, early fibreoptic bronchoscopy is recommended for any immunocompromised patient with new onset of hoarseness, unexplained sore throat, or signs of airway obstruction. The bronchoscopic features described should alert clinicians to invasive aspergillosis as an underlying diagnosis, and antifungal therapy should be started as soon as possible.

1 Fertilo A. Primary aspergillosis of the larynx. F Laryngol Otol 1974;88:1257-63.

2 Kheir SM, Flint A, Moss JA. Primary aspergillosis of the larynx simulating carcinoma. Hum Pathol 1983;14:184-6.

3 Benson-Mitchell R, Croft CB, Gallimore A. Aspergillosis of the larynx. F Laryngol Otol 1994;108:883-5.

4 Balsam D, Sorrano D, Barax C. Candida epiglottitis presenting as stridor in a child with HIV infection. Pediatr Radiol 1992;22:235-6.

5 Peterson PK, McGlave P, Ramsay NK. A prospective study of infectious diseases following bone marrow transplantation: emergence of Aspergillus and cytomegalovirus as the major causes of mortality. Infect Control 1983;4:81-9.

6 Berlinger NT, Freeman TJ. Acute airway obstruction due to necrotizing tracheobronchial aspergillosis in immunocompromised patients: a new clinical entity. Ann Otol Rhinol Laryngol 1989;98:718-20.

7 Kemper CA, Hostetler JS, Follansbee SE, Ruane P, Covington D, Leong SS, et al. Ulcerative and plaque-like tracheobronchitis due to infection with Aspergillus in patients with AIDS. Clin Infect Dis 1993;17:344-52.

8 Kramer MR, Denning DW, Marshall SE, Ross DJ, Berry G, Lewiston NJ, et al. Ulcerative tracheobronchitis after lung transplantation. A new form of invasive aspergillosis. $\mathrm{Am}$ transplantation. A new form of
Rev Respir Dis 1991;144:552-6.

9 Young RC, Bennett JE, Vogel CL, Carbone PP, DeVita VT. Aspergillosis. The spectrum of the disease in 98 patients. Medicine 1970;49:147-73.

10 Denning DW, Follansbee SE, Scolaro M, Norris A, Edelstein H, Stevens DA. Pulmonary aspergillosis in acquired immunodeficiency syndrome. $N \mathrm{Engl} F \mathrm{~F}$ Med 1991;324:654-62. 